\title{
Exploration of retinoblastoma pathogenesis with bioinformatics
}

\author{
Ying Zhang, Li Zhou, Shan Wang, Man Wang, Shangchao Wu \\ Department of Ophthalmology, Wuhan Aier Eye Hospital of Wuhan University, Wuhan, China \\ Contributions: (I) Conception and design: S Wu, Y Zhang; (II) Administrative support: S Wu; (III) Provision of study materials or patients: Y Zhang, \\ L Zhou; (IV) Collection and assembly of data: S Wang, M Wang; (V) Data analysis and interpretation: S Wu, Y Zhang; (VI) Manuscript writing: All \\ authors; (VII) Final approval of manuscript: All authors. \\ Correspondence to: Shangchao Wu. Department of Ophthalmology, Wuhan Aier Eye Hospital of Wuhan University, Wuhan 430000, China. \\ Email: wushchwhae@sina.com.
}

Background: Differentially expressed genes (DEGs) from retinoblastoma (RB) tissues play key roles in the progression of RB. However, the role of DEGs in different subtypes and stages of RB has not yet been systematically analyzed.

Methods: In this study, the DEGs for tumor and adjacent from 3 RB data sets GSE24673, GSE97508, and GSE110811 were analyzed with regard to the different subtypes and stages of the disease.

Results: Through comparison with adjacent tissues, a total of 78 upregulated genes and 155 downregulated genes from the RB tissues were identified across the 3 data sets. Gene set enrichment analysis (GSEA) showed that the 3 representative genes $C D K 1, C D C 20$, and $B U B 1$, which were all upregulated, could promote the cell cycle in RB. Compared with adjacent tissues in GSE97508, a total of 19 gigantol-targeted genes were predicted to be upregulated in invasive RB tissues. On the other hand, DEGs for tumor and adjacent from 3 RB data sets GSE24673, GSE97508, and GSE110811 were integrated with regard to invasiveness and stages of the disease, and another 19 DEGs were subsequently identified. Among these genes, UHRF1 was the only identified upregulated gene, while the other 18 were all downregulated genes. Cell Counting Kit-8 (CCK8) experiment and GSEA results showed that UHRF1 can promote the proliferation and invasion of RB. Conversely, the downregulated representative gene CADM1 is a tumor suppressor gene, which can inhibit the progression of RB.

Conclusions: This study indicated that the verified DEGs are continuously and consistently expressed in different subtypes and stages of RB. These DEGs may be the key to understanding the development and invasion of RB.

Keywords: Differentially expressed genes (DEGs); gene set enrichment analysis (GSEA); invasion; proliferation; retinoblastoma $(\mathrm{RB})$

Submitted May 31, 2021. Accepted for publication Jul 14, 2021.

doi: $10.21037 /$ tcr-21-1034

View this article at: https://dx.doi.org/10.21037/tcr-21-1034

\section{Introduction}

Retinoblastoma $(\mathrm{RB})$ is a retinal embryonic malignant tumor common in infants and young children (1), and is also the most common primary malignant retinal tumor (2). The global incidence of RB is 1 in 20,000, accounting for $4 \%$ of all cancer types in children. The incidence of $\mathrm{RB}$ is relatively high, with nearly 8000 newborns suffering from RB worldwide each year (3). Not being diagnosed and treated in time is considered a fundamental constraint for $\mathrm{RB}$ patients in the developing world. In the past few years, treatment of RB has significantly improved, and the survival rate of patients has been increasing (4,5). Despite this, RB still poses a huge threat to the health of children. The use of standard chemotherapy regimens may cause a series of adverse reactions in patients. In addition, intracranial and distant metastases often endanger the lives of children with RB. Therefore, researchers are trying to clarify the 
molecular mechanism for the occurrence and development of RB so that new molecular therapeutic targets can be found to ameliorate the management of $\mathrm{RB}$ and improve the quality of life of RB patients.

Differentially expressed genes (DEGs) between $\mathrm{RB}$ tissues and adjacent tissues are likely the key to understanding the pathology of RB (2). Similarly, the DEGs between various disease stages and subtypes could clarify the mechanism underlying RB progression. However, DEGs for different disease stages and subtypes have not been well studied in RB.

Surgery, systemic radiotherapy, and chemotherapy all have certain side effects. Currently, the management of RB is not satisfactory, and the quality of life of patients should be further improved (5). In recent years, traditional Chinese medicine (TCM) has attracted much attention due to its wide range of sources and low side effects and has become a focal point in the research of antitumor drugs (6). Network pharmacology, which involves the search for drug targets in databases and constructing target-target and target-pathway interactions, has become a convenient method for exploring TCM pharmacology; thus, it has grown rapidly as a subfield (7). Gigantol, a double benzyl compound, is one of more the common polyphenols found in TCM. Increasing numbers of studies have shown that gigantol produces anticancer effects $(8,9)$. However, the role and mechanism of gigantol in RB has not yet been reported.

In this study, we analyzed the DEGs in adjacent and RB tissues from multiple data sets. Gene Ontology (GO) and Kyoto Encyclopedia of Genes (KEGG) enrichment was applied. Enrichment and analysis were also applied in DEGs for mild, moderate and late-stage tissues, as well as invasive and adjacent tissues. In addition, in order to clarify the potential mechanism of gigantol action in $\mathrm{RB}$, we preliminarily explored its effect using network pharmacology. Finally, the shared DEGs in the above analysis were identified. These genes may be new targets for $\mathrm{RB}$ diagnosis and intervention.

We present the following article in accordance with the MDAR reporting checklist (available at https://dx.doi. org/10.21037/tcr-21-1034).

\section{Methods}

\section{Microarray data sets}

The Gene Expression Omnibus (GEO) (http://www.ncbi. nlm.nih.gov/geo) is a public genomics database of the
National Center for Biotechnology Information Company (NCBI), which stores high-throughput gene expression data and microarray data. The RB-related microarray data sets GSE97508, GSE24673, and GSE110811 were used to analyze the DEGs in the RB tissues.

The study was conducted in accordance with the Declaration of Helsinki (as revised in 2013)

\section{DEG analysis}

The GEO2R online processing tool (http://www.ncbi.nlm. nih.gov/geo/geo2r) was used to analyze the DEGs of each data set in the GEO database. The screening criteria were $|\log \mathrm{FC}| \geq 1$ and $\mathrm{P}<0.05$.

\section{GO and KEGG enrichment analysis}

The functional annotation and pathway enrichment of DEGs was applied. The Search Tool for the Retrieval of Interacting Genes/Proteins (STRING) online database (https://www.string-db.org/) was used for GO and KEGG enrichment analysis. All enrichment analyses were based on Homo sapiens. A false discovery rate (FDR) of $<0.05$ was considered statistically significant.

\section{Protein-protein interaction (PPI) (network construction)}

The interactions between DEGs were confirmed using the STRING online database for Homo sapiens, and a PPI network of DEGs was constructed and visualized by Cytoscape.

\section{Gene set enrichment analysis (GSEA)}

The GSE97508, GSE24673, and GSE110811 gene sets were imported into GSEA 4.0 software, and GSEA analysis was performed by the software's default parameters. An FDR of $<0.25$ was considered to be significantly enriched. GSE110811's data matrix (https://www.ncbi.nlm.nih.gov/ geo/download/?acc=GSE110811), cell cycle and invasionrelated gene sets (http://www.gsea-msigdb.org/) were imported. The RB tissues were divided into high or low expression groups according to the median levels of the CDK1, CDC20, BUB1, UHRF1, and CADM1 genes in the RB tissues. GSEA was run to analyze the relationship between the levels of $C D K 1, C D C 20, B U B 1$, and cell proliferation; and the relationship between levels of $U H R F 1$ and $C A D M 1$ and cell invasion. 


\section{Gigantol target prediction}

SwissTargetPrediciton (taking the first 15 genes) and TargetNet (pro $>0$ ) were used to predict the targets of gigantol. The results of these 2 analyses combined was considered to be the predictive target of gigantol.

\section{Plasmid construction and cell culture}

UHRF1 and CADM1 plasmids were constructed by amplifying the prokaryotic expression vector and the plasmids were then connected to the pcDNA3.1 vector. The sequence was verified by polymerase chain reaction (PCR) amplification. Human RB cell lines, Y79 and SO-RB50, were purchased from Fuheng Biotechnology (Shanghai, China). The cells were grown in an RPMI-1640 medium containing $10 \%$ fetal bovine serum and $1 \%$ streptomycin/ penicillin, under culture conditions of a $37^{\circ} \mathrm{C}, 5 \%$ carbon dioxide atmosphere. Lipofectamine LTX Plus reagent (Invitrogen, Carlsbad, CA, USA) was used for transfection experiments. Gigantol (809AKR1B101) was acquired from ProSpec-Tany TechnoGene Ltd. (Ness-Ziona, Israel).

\section{Western blotting experiment}

The protein samples extracted from the cells were separated with $10 \%$ sodium dodecyl sulfate (SDS)-polyacrylamide gel electrophoresis (PAGE) and then transferred to a polyvinylidene fluoride (PVDF) membrane. The samples were then blocked with tris-buffered saline and Tween 20 (TBST) with $5 \%$ skimmed milk at room temperature for 1 hour, and then the primary antibody was incubated in a refrigerator at $4{ }^{\circ} \mathrm{C}$ (dilution ratio of $1: 1,000$ ) overnight. The membrane was washed with TBST buffer solution 4 times for 5 minutes. According to the instructions of the primary and secondary antibodies, the membrane was incubated with horseradish peroxidase (HRP)-linked anti-rabbit immunoglobin G (IgG) secondary antibody $(1: 3,000)$ for 1 hour at room temperature and washed 4 times with TBST, with exposure being performed using the electrochemiluminescence (ECL) method. All primary antibodies were acquired from ABclonal Technology (Beijing, China), and the secondary antibody was acquired from Zhongshan Golden Bridge Technology Company (Beijing, China).

\section{Cell proliferation}

Approximately 3,000 cells were seeded into each well of a 96-well plate, with 6 replicated wells for each group. After the cells adhered to the wall, a Cell Counting Kit-8 (CCK-8) kit (Tongren, Kumamoto, Japan) was added to test the cell viability. For paralleled plates, viability was tested every 24 hours for 5 consecutive days. Data were collected with a microplate reader.

\section{Statistical analysis}

The in vitro experiment was biologically repeated 3 times. One-way analysis of variance (ANOVA) or $t$-test was used to compare the differences between each group.

\section{Results}

\section{The DEG profile of $R B$ and adjacent tissues}

To better understand the pathology of RB, 3 RB data sets were retrieved, and analysis of DEGs was performed between adjacent tissues and RB tissues. Compared to adjacent tissues, the RB tissues showed 2,008 upregulated genes and 3,228 downregulated genes in the GSE97508 data set, 861 upregulated and 2,553 downregulated genes in the GSE24673 data set, and 194 upregulated genes and 242 downregulated genes in the GSE110811 data set (Figure 1A). Figure 1B,C respectively show the number of these differential genes and the overlapping relationships between them. It is worth noting that 78 genes were coupregulated and 155 genes (233 in total) were codownregulated across the 3 data sets. GO analysis of 78 coupregulated genes was performed to study their potential biological functions. Biological process (BP) analysis results show that the upregulated genes were significantly enriched in the cell cycle, which may be the cause of the accelerated cell cycle of RB (Figure 1D). In addition, GO $\mathrm{BP}$ enrichment analysis was performed on 155 common downregulated genes, and the results showed that the downregulated genes were most significantly enriched in signal transduction and cell communication (Figure 1E), which is consistent with the biological characteristics of RB cells.

\section{DEGs in RB can form abundant PPIs to regulate the cell cycle}

In order to further understand the role of the DEGs in the development of RB, the STRING online analysis tool was used to perform PPI analysis on the 233 DEGs mentioned 

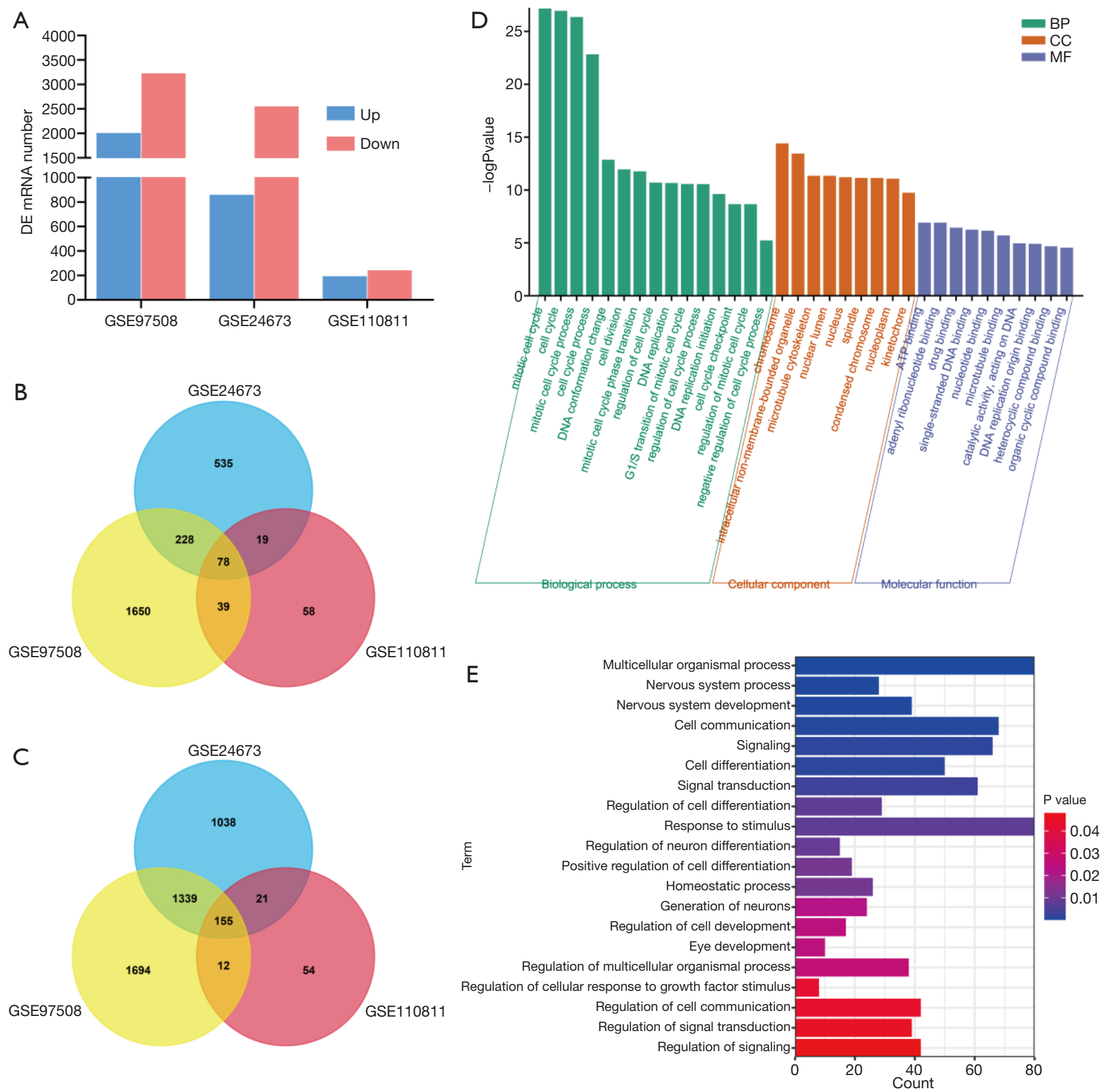

Figure 1 Differentially expressed gene (DEG) and Gene Ontology (GO) analysis in the retinoblastoma (RB) tissues. (A) The number of DEGs for the adjacent and RB tissues in the GSE97508, GSE24673, and GSE110811 data sets. Venn diagram showing the number of upregulated (B) and downregulated (C) DEGs and their overlapping relationship. (D) GO enrichment results of the 78 upregulated DEGs. (E) GO BP enrichment results of the 155 downregulated DEGs. mRNA, messenger RNA.

above. A PPI network composed of 47 node proteins was obtained, with each node representing 1 gene and lines representing interactions between proteins (Figure $2 A$ ). The interacting proteins were then imported into Cytoscape for visualization. Blue represents upregulated genes, and red represents downregulated genes. The most frequently 
connected key genes in the network were $C D K 1, C D C 20$, and $B U B 1$. GSEA analysis was performed in order to better understand the role of these 3 key genes in RB. The results showed that the cell cycle-related gene sets were significantly enriched in the CDK1 (Figure 2B), CDC20 (Figure 2C), and BUB1 (Figure 2D) high-expression samples. The above results indicated that the high expression of these 3 genes may promote cell cycle and thus tumor progression in $\mathrm{RB}$.

\section{$R B$ tissues from different stages showed distinct messenger $R N A$ expression profiles}

The GSE110811 data from RB tissues of different subtypes were collected. GEO2R was used to obtain DEGs from these different subtypes. Compared to the adjacent tissues, there were 63 upregulated genes and 159 down-regulated genes in the mild RB tissues, 17 upregulated genes and 245 downregulated genes in the moderate RB tissues, and 194 upregulated genes and 162 downregulated genes in the severe RB tissues (Figure 3A). A Venn diagram was drawn, which showed the number of DEGs and the overlapping relationship between them. Five up-regulated genes and 48 down-regulated genes (53 in total) were identified in the mild, moderate, and severe RB samples (Figure 3B,C). Next, GO BP/cellular component (CC) analysis was performed on these 53 DEGs. In Figure 3D, the right-hand lanes represent $\mathrm{BP}$ analysis, and the left-hand lanes represent $\mathrm{CC}$ analysis. BP analysis showed that nervous system processes, visual perception, and maintenance of photoreceptor cells were the main enriched processes. The main enriched CCs included the plasma membrane area, photoreceptor, and ciliary body membrane. The GO chord diagram further showed that RHO, CNGA1, PCDH15, and RDH12 are involved in multiple biological processes (Figure 3E).

\section{Invasive RB tissues showed distinct messenger $R N A$ expression profiles}

The GSE97508 data from invasive and noninvasive $\mathrm{RB}$ tissues and adjacent tissues were collected. GEO2R analysis showed that compared to the adjacent tissues, 8,718 upregulated genes and 6,415 down-regulated genes were present in the invasive the $\mathrm{RB}$ tissues (Figure 4A). Gigantol is a main active ingredient extracted and isolated from the orchid Dendrobium draconis, which is used in TCM. Several studies have shown that gigantol has antitumor effects, which are mainly exerted through inhibiting the viability and mobility of the tumor $(10,11)$. Therefore, intersections of the predicted genes targeted by gigantol, the DEGs in tumors and adjacent tissues, and the upregulated DEGs in adjacent tissues and invasive RB tissues from the GSE97508 data set were generated into a subset of 19 genes (Figure 4B). GO and KEGG enrichment analysis were performed on these 19 genes. The main BP included signal transduction, cell communication, regulation of cell population proliferation, and response to drugs. The most involved CCs were cell periphery, plasma membrane, and synapse. Consistent with the foregoing results, the key molecular functions (MFs) involved were signal receptor activity, $G$ protein-coupled receptor activity, and neurotransmitter: sodium symporter activity (Figure 4C). KEGG analysis showed that signaling pathways related with these 19 genes were mainly interaction of neuroactive ligand receptors, calcium signaling, and AMPK signaling, all of which are closely related to tumor occurrence and development (Figure 4D). Figure 4E shows the levels of these 19 genes in the RB tissues. The CCK8 results showed that the proliferation of $\mathrm{Y} 79$ and SORB50 cells was significantly inhibited after different concentrations of gigantol treatment (Figure 4F,G). ESR1 and TERT with relatively small $\log \mathrm{FC}$ values, and NOS2 and $C A 9$ with relatively large $\log \mathrm{FC}$ values, were selected to confirm the relationship with gigantol. As expected, the western blotting results showed that gigantol could inhibit the expression of these 4 proteins in a concentrationdependent manner in RB cell lines (Figure 4H).

\section{UHRF1 and CADM1 could regulate the proliferation of RB cells}

The 78 genes that were upregulated in the RB tissues from all 3 data sets; the 8,606 genes that were upregulated in the invasive RB tissues from the GSE97508 data set; and the $5 \mathrm{u}$-regulated genes identified in the mild, moderate, and severe RB samples from GSE110811 were integrated (Figure 5A). UHRF1 was identified as the only shared upregulated gene. Similarly, the downregulated DEGs for these subtypes were analyzed, and 18 downregulated genes were identified as shared downregulated genes for all subtypes (Figure 5B). Figure $5 C$ shows the $\log \mathrm{FC}$ values of the 18 downregulated genes in different data sets. The Y79 and SO-RB50 cell lines that stably overexpressed UHRF1 or CADM1 were constructed and confirmed with western blotting (Figure 5D). The CCK8 results showed that, consistent with the previous results, UHRF1 

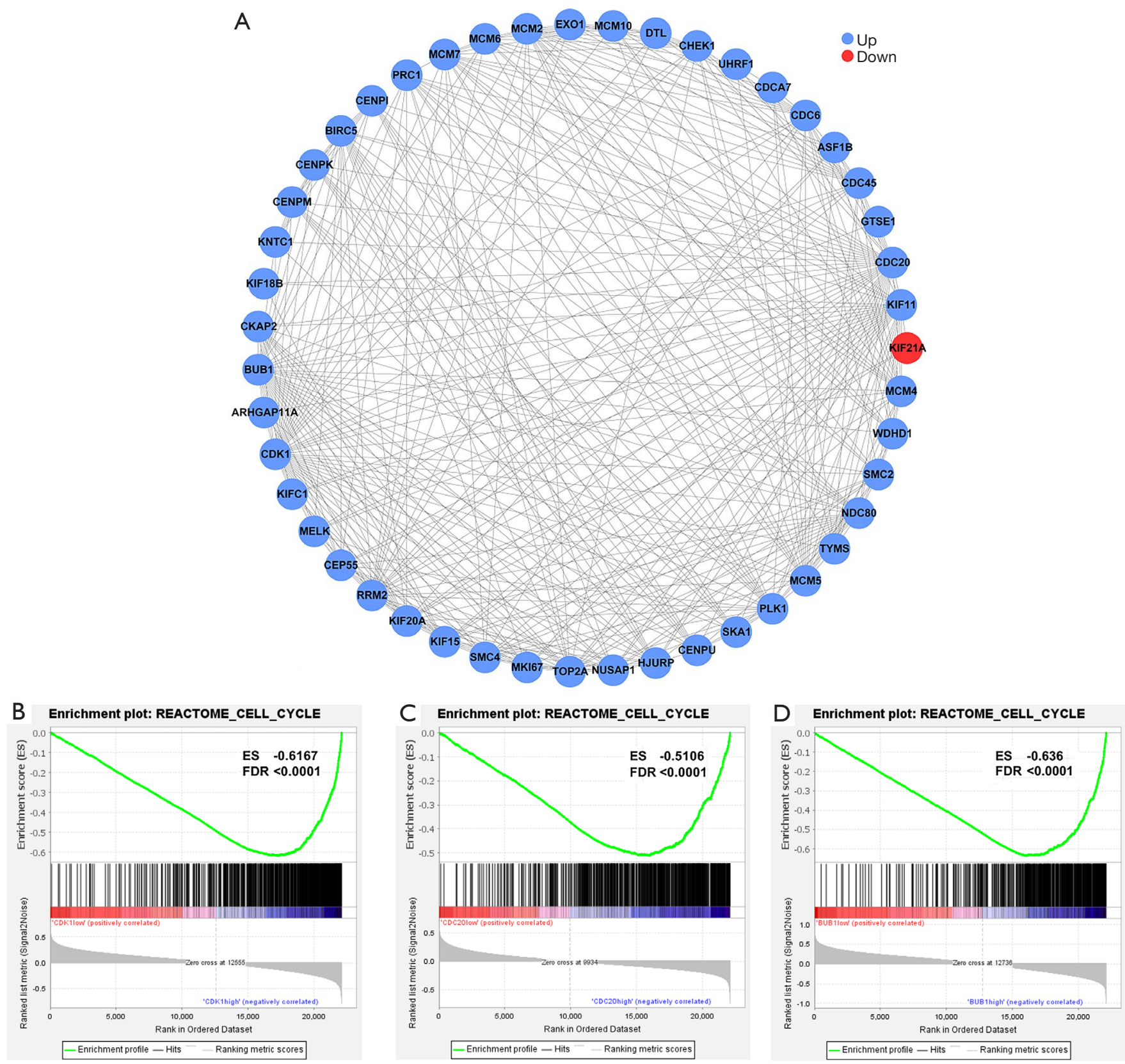

Figure 2 Protein-protein interaction (PPI) and gene set enrichment analysis (GSEA) analysis of differentially expressed genes (DEGs) indicating correlation with the cell cycle. (A) PPI network of DEGs. The color of the node indicates the level of expression. GSEA analysis of $C D K 1(\mathrm{~B}), C D C 20(\mathrm{C})$, and $B U B 1$ (D) in retinoblastoma (RB). The high and low expression group for each gene is demarcated with the median expression levels of $C D K 1, C D C 20$, and $B U B 1$.

could significantly promote the proliferation of RB cells, while $C A D M 1$ could inhibit the proliferation of RB cells (Figure $5 E, F, G, H)$. Similar with the above results, GSEA analysis results show that $U H R F 1$ could promote cell invasion and that CADM1 could inhibit cell invasion (Figure 5I,7).

\section{Discussion}

In this study, tumor and adjacent tissues from $3 \mathrm{RB}$ data sets, GSE97508, GSE24673, and GSE110811, were examined by GEO2R analysis, with 78 coupregulated 

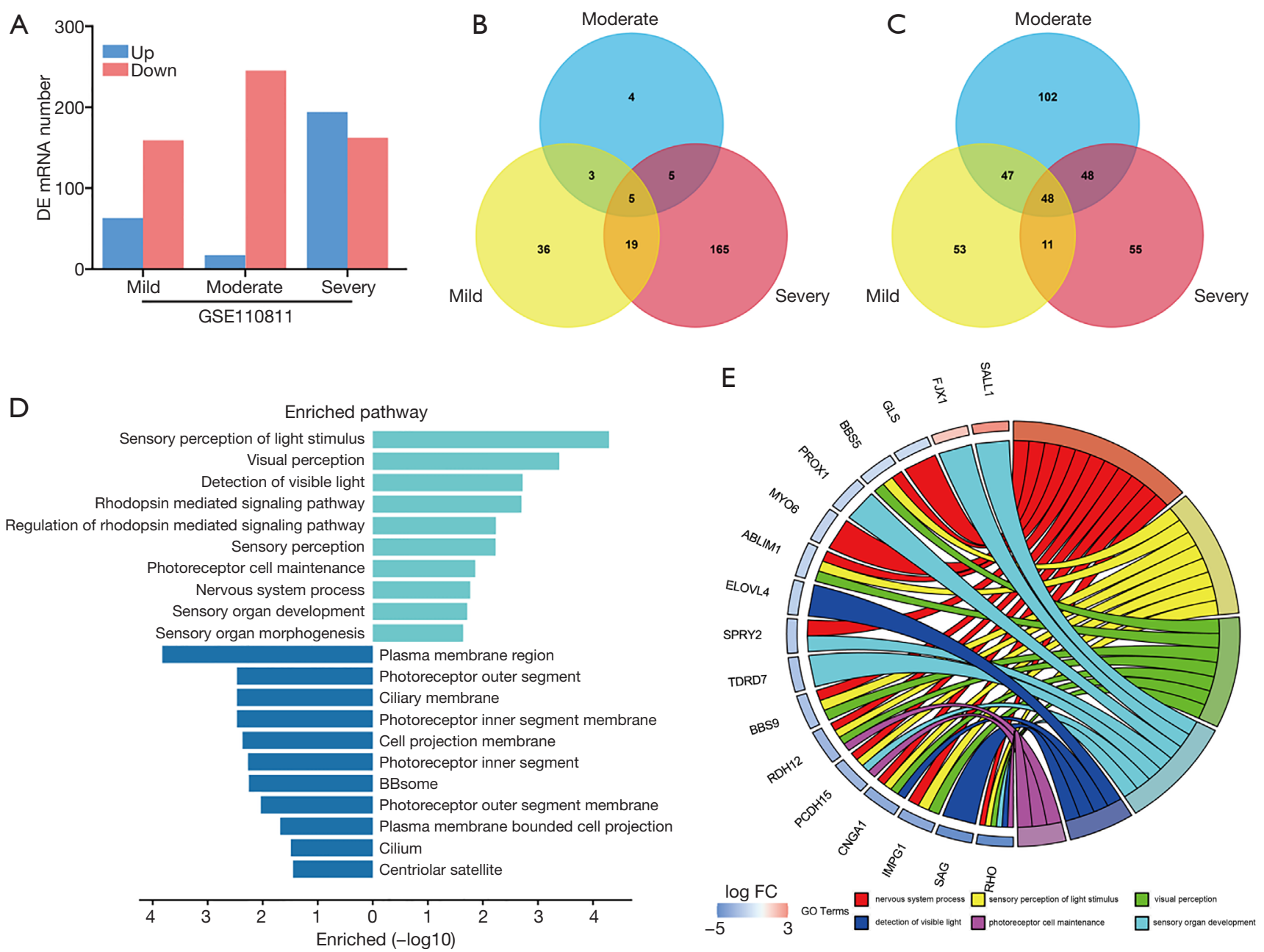

Figure 3 Differentially expressed genes (DEGs) and Gene Ontology (GO) enrichment results in anaplastic retinoblastoma (RB) tissue samples. (A) The number of DEGs for adjacent tissues, and mild, moderate, and severe RB tissues. The number of upregulated (B) and downregulated (C) DEGs and their overlapping relationship. (D) GO analysis of the 53 overlapping DEGs. Right-hand lanes represent biological process (BP) analysis, and left-hand lanes represent cell component (CC) analysis. (E) GO chord diagram based on GO analysis, showing the overlapping DEGs observed in the main BP components. mRNA, messenger RNA.

genes and 155 codownregulated genes being identified. GO enrichment analysis of DEGs suggests that cell cycle changes are important features of RB. Cell cycle is an evolutionarily conservative process necessary for the growth and development of mammalian cells, and abnormal cell cycle regulation is a sign of tumor $(12,13)$. Our enrichment results accorded with these facts. In addition, the 3 genes, $C D K 1, C D C 20$, and $B U B 1$ were found to be the key genes in the PPI network. CDK1 is mainly responsible for promoting G1/S phase and G2/M switching (14). Previous studies have also shown that CDK1 is abnormally expressed in a variety of tumors $(15,16)$. Based on the GSEA method, the relationship between the expression level of CDK1 in the RB tissues and the cell cycle was analyzed. The positive regulatory cell cycle-related gene set was significantly enriched in the CDK1 high expression group; that is, $C D K 1$ promoted the cell cycle in RB tissues. Similarly, two other closely related genes in the cell cycle, $C D C 20$ and $B U B 1$, were also found to promote the cell cycle. These results suggest that $C D K 1, C D C 20$, and $B U B 1$ may serve as potential new targets for RB therapy.

Most DEGs identified compared gene expression between tumor tissues and adjacent tissues across data sets in RB-related research. However, previous reports 

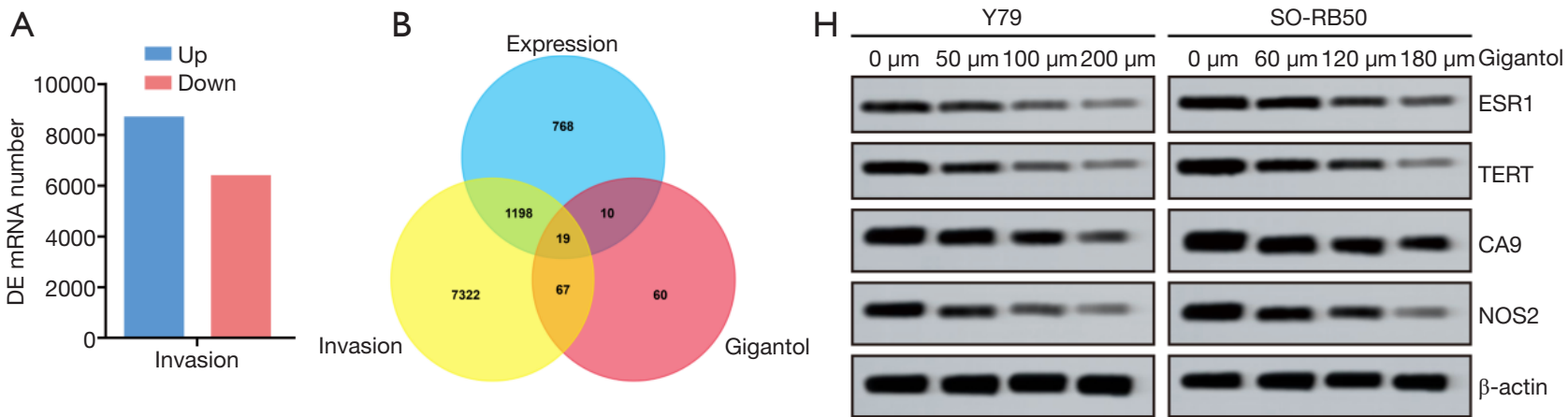
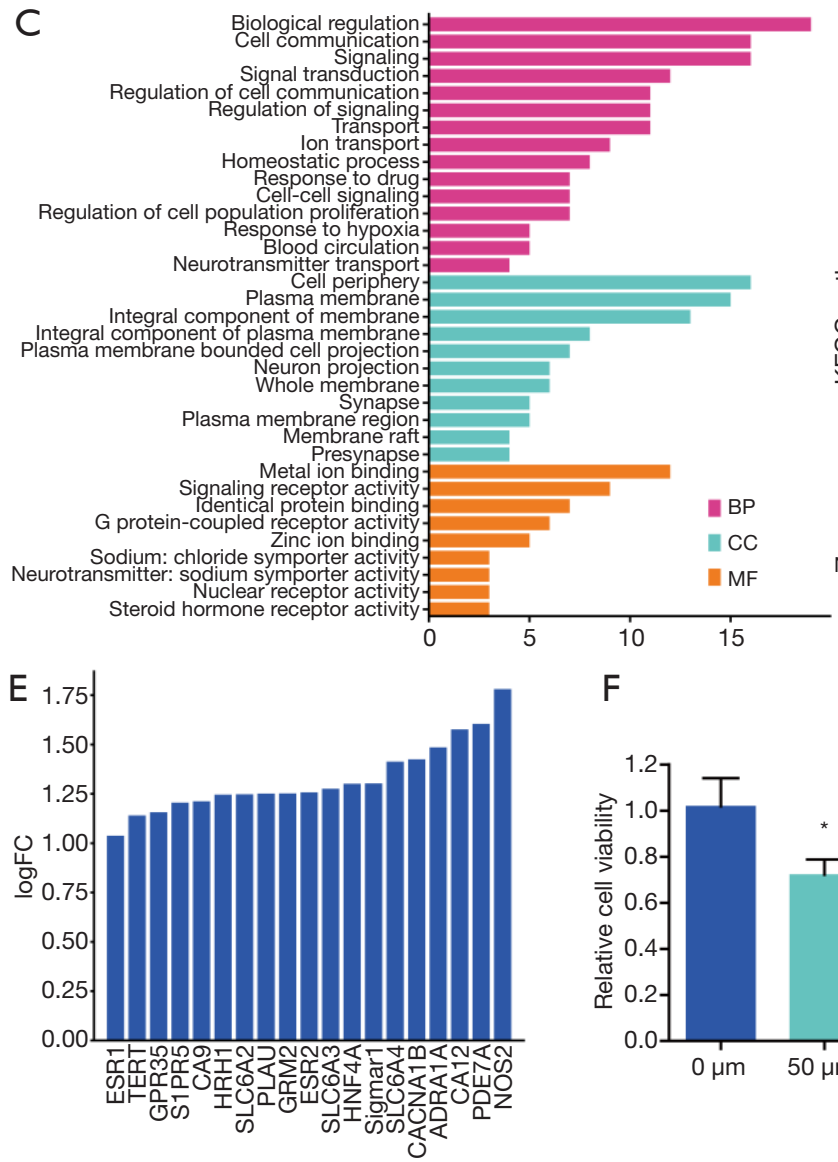

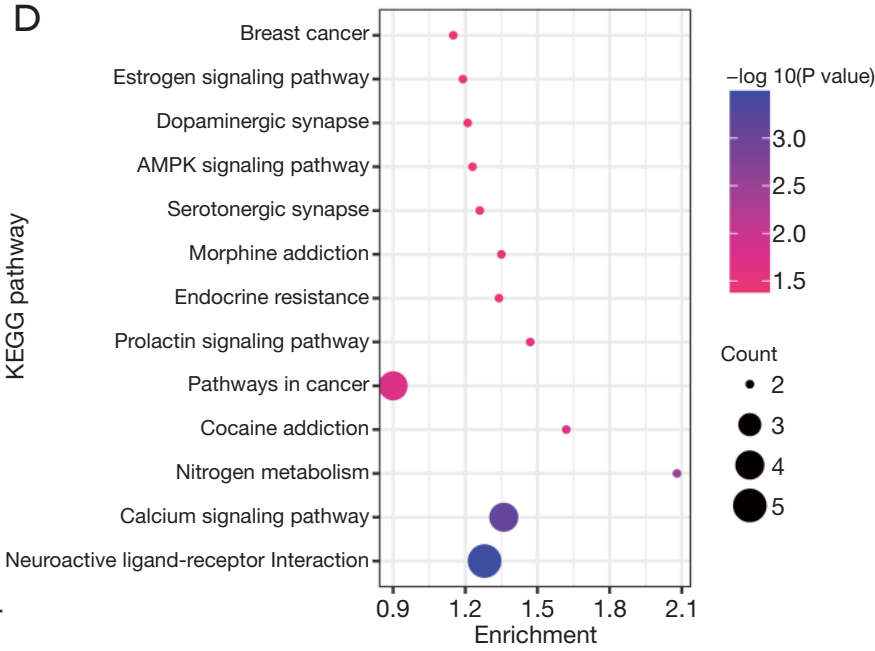

G
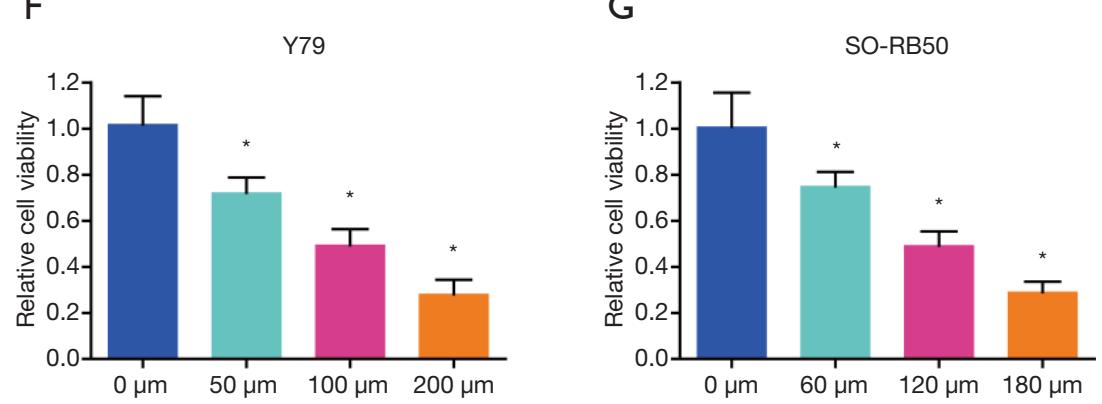

Figure 4 Overlap of gigantol targets and retinoblastoma (RB) differentially expressed genes (DEGs), and the anti-RB effects of gigantol. (A) The number of DEGs for adjacent and invasive RB tissues in the GSE97508 data set. (B) The number of DEGs and the overlapping relationship of DEGs for adjacent and invasive RB tissues, gigantol-targeted genes, and DEGs that were upregulated in the GSE97508 data set. Gene Ontology (GO) (C) and Kyoto Encyclopedia of Genes (KEGG) (D) analysis results of the 19 overlapping genes. (E) The logFC value of 19 genes. (F,G) CCK8 experiment results show that gigantol can inhibit the viability of RB cells. (H) Western blotting results suggest that gigantol inhibits the expression of the overlapping genes. ${ }^{*} \mathrm{P}<0.05$ versus $0 \mu \mathrm{m}$ group. mRNA, messenger RNA.

show that not all DEGs are continuously and consistently differentially expressed in different tumor stages (17). Therefore, in this study, the GSE110811 data set containing different stages of the RB tissues was analyzed. We found
5 upregulated DEGs and 48 downregulated DEGs for different stages. GO enrichment results suggested that RHO, PCDH15, RDH12 and other genes are involved in multiple biological processes closely related to tumor 
A

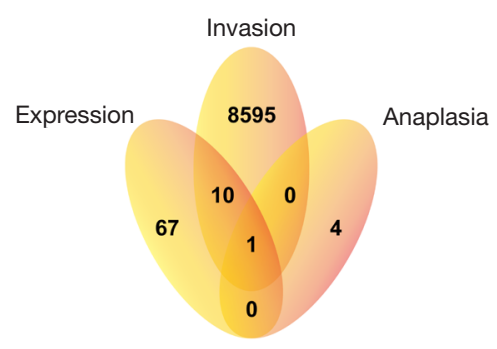

D GSE97508
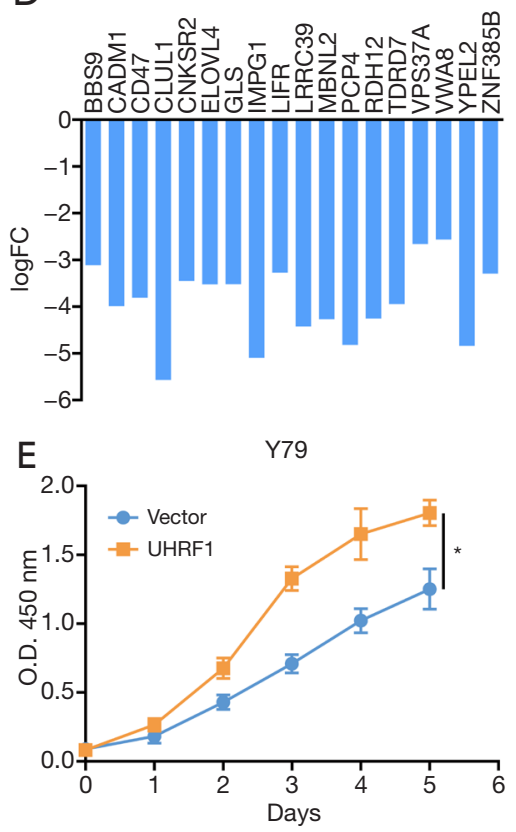

$\mathrm{H}$

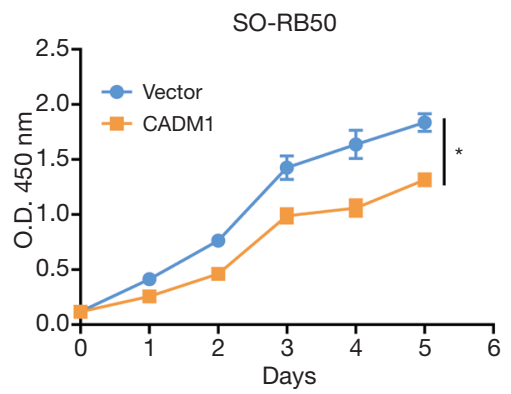

B

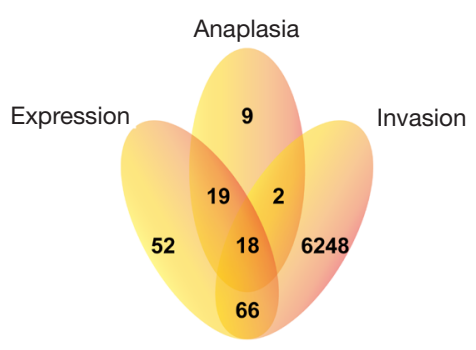

GSE24673

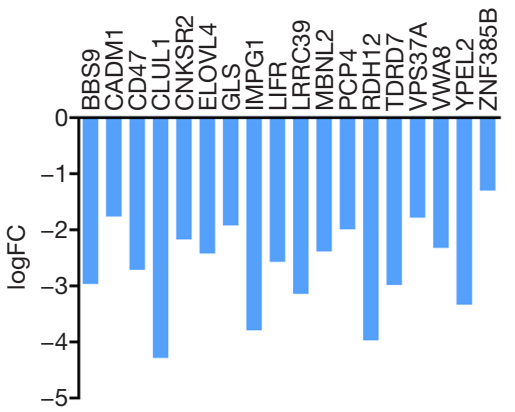

$\mathrm{F}$
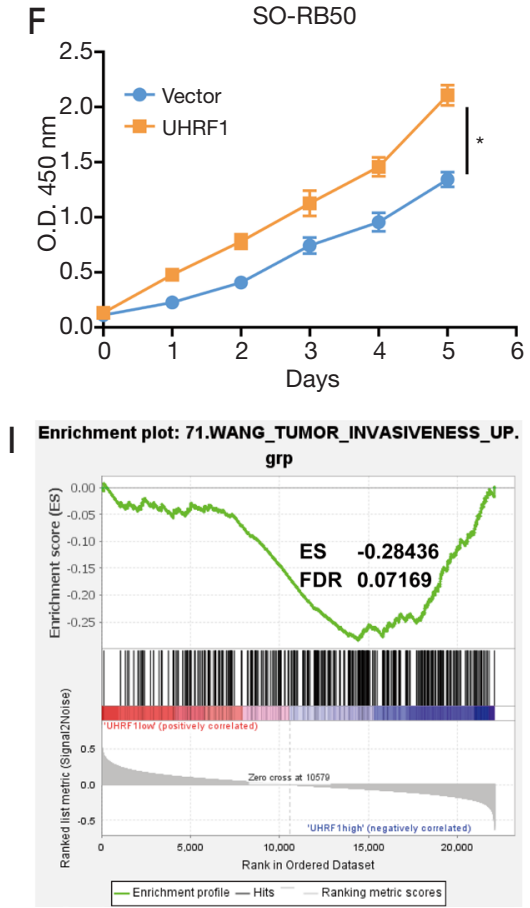

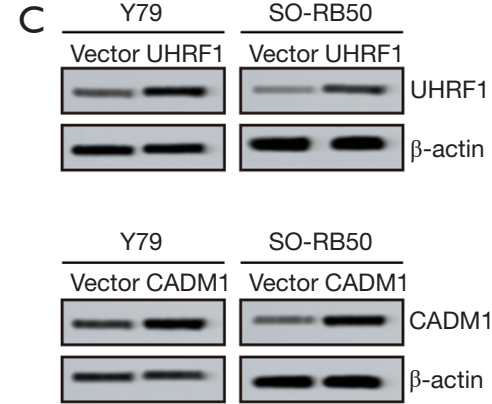

GSE110811

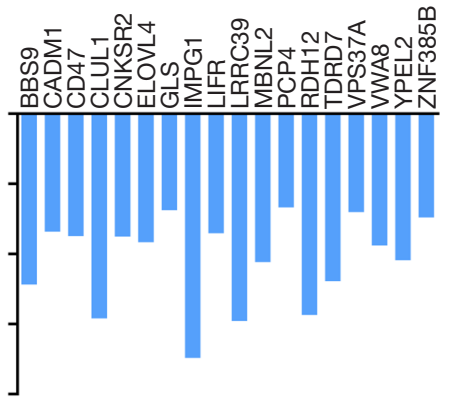

G

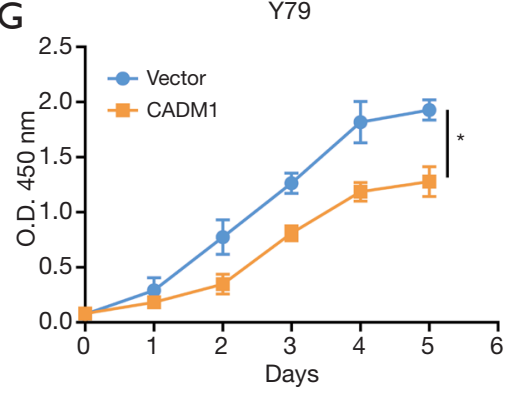

Enrichment plot: 72.
ANASTASSIOU_MULTICANCER_INVASIVENESS_SIGNAT

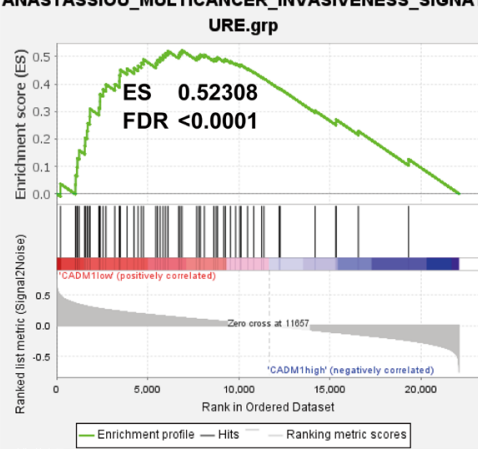

Figure 5 UHRF1 and CADM1 regulate the proliferation of retinoblastoma (RB) cells. The number of upregulated (A) and downregulated (B) DEGs and their overlapping relationship for differentially expressed genes (DEGs) of adjacent and retinoblastoma (RB) tissues in all 3 data sets, adjacent, and invasive RB tissues in GSE97508, and the DEGs identified in the mild, moderate, and severe RB samples from GSE110811. (C) The $\operatorname{logFC}$ values of the 18 downregulated DEGs in the 3 data sets. (D) Western blotting confirmed the overexpression of UHFR1 and CADM1 in Y79 and SO-RB50 cells. Viability of UHFR1-overexpressed Y79 (E) and SO-RB50 (F) cells tested with Cell Counting Kit-8 (CCK-8). Viability of CADM1-overexpressed Y79 (G) and SO-RB50 (H) cells tested with CCK-8. Gene set enrichment analysis (GSEA) analysis of UHFR1 (I) and CADM1 (J) in RB was applied. The high and low expression group for each gene is demarcated with the median expression levels of UHFR1 and CADM1. ${ }^{*} \mathrm{P}<0.05$ versus Vector group. 
progression. Previous studies have shown that the activation of the RHO signaling pathway is closely related to the malignant phenotype of tumors (18), which supports the reliability of our work.

Additionally, DEGs from invasive RB and adjacent tissues were identified. Gigantol has been reported to have antitumor effects $(9,10)$, but its role in RB is yet to be reported. In this study, we found that the predicted 19 targets of gigantol were upregulated in both the RB tissues, especially those of invasive RB. CCK- 8 results showed that gigantol can inhibit the viability of RB cells. It was further confirmed that gigantol inhibited the protein expression of ESR1, TERT, and CA9, all of which have relatively small $\log \mathrm{FC}$ values. The expression of NOS2, which has a large $\log \mathrm{FC}$ value, was also inhibited by gigantol. The results suggested that these 19 genes may be the targets of gigantol, and their high expression could promote the invasion of RB.

Finally, DEGs for tumor and adjacent tissues, invasive and adjacent tissues in GSE97508, and adjacent tissues from different stages in GSE110811 were integrated. UHRF1 was identified as the only DEG that was upregulated. On the other hand, there were 18 downregulated DEGs. It was further confirmed that $U H R F 1$ promotes the proliferation and invasion of RB cells. On the contrary, CADM1, as a representative of 18 downregulated DEGs, was found to negatively regulate the phenotype of RB cells. Several previous studies have shown that $U H R F 1$ is related to the occurrence and development of RB $(19,20)$. This study reveals the key role of $U H R F 1$ in $\mathrm{RB}$ from the perspective of bioinformatics, further supporting $U H R F 1$ as a potential diagnostic and therapeutic biomarker for RB. CADM1 has been reported to be downregulated in a variety of tumors and to inhibit the progression of ovarian cancer, breast cancer, liver cancer, and other tumors (21-23). However, its role in RB has not yet been reported. Our results found evidence for the tumor-suppressing effect of $C A D M 1$ in $\mathrm{RB}$ and further suggested the possibility of CADM1 as a tumor suppressor.

In summary, our study identified DEGs for tumor and adjacent from 3 RB data sets GSE24673, GSE97508, and GSE110811 with regard to invasiveness and stages of the disease. The results revealed that $C D K 1, C D C 20$, and $B U B 1$ are upregulated in $\mathrm{RB}$ and participate in the process of $\mathrm{RB}$ by promoting the cell cycle. The pharmacological effects of gigantol in treating $\mathrm{RB}$ were briefly characterized. The role of $U H R F 1$ in RB was confirmed with bioinformatics, further suggesting that $U H R F 1$ could be a potential diagnostic and therapeutic target for RB. The roles of the key DEGs in RB were confirmed with related overexpression vectors in $\mathrm{RB}$ cell lines Y79 and SO-RB50. CADM1 is downregulated in $\mathrm{RB}$, which possibly increases the proliferation and invasion of RB cells. This study reveals new mechanisms for the development and invasion of RB. However, the main body of DEGs observed in this study still need to be investigated further in future research.

\section{Acknowledgments}

Funding: None.

\section{Footnote}

Reporting Checklist: The authors have completed the MDAR reporting checklist. Available at https://dx.doi. org/10.21037/tcr-21-1034

Conflicts of Interest: All authors have completed the ICMJE uniform disclosure form (available at https://dx.doi. org/10.21037/tcr-21-1034). The authors have no conflicts of interest to declare.

Ethical Statement: The authors are accountable for all aspects of the work in ensuring that questions related to the accuracy or integrity of any part of the work are appropriately investigated and resolved. The study was conducted in accordance with the Declaration of Helsinki (as revised in 2013). Institutional ethical approval and informed consent were waived.

Open Access Statement: This is an Open Access article distributed in accordance with the Creative Commons Attribution-NonCommercial-NoDerivs 4.0 International License (CC BY-NC-ND 4.0), which permits the noncommercial replication and distribution of the article with the strict proviso that no changes or edits are made and the original work is properly cited (including links to both the formal publication through the relevant DOI and the license). See: https://creativecommons.org/licenses/by-nc-nd/4.0/.

\section{References}

1. Siegel RL, Miller KD, Jemal A. Cancer statistics, 2020. CA Cancer J Clin 2020;70:7-30.

2. Huang J, Zhang L, Li Z, et al. Screening and identification of key biomarkers for retinoblastoma: Evidence from bioinformatics analysis. Medicine (Baltimore) 
2020;99:e19952.

3. Global Retinoblastoma Study Group; Fabian ID, Abdallah E, et al. Global Retinoblastoma Presentation and Analysis by National Income Level. JAMA Oncol 2020;6:685-95.

4. Tomar AS, Finger PT, Gallie B, et al. Global

Retinoblastoma Treatment Outcomes: Association with National Income Level. Ophthalmology 2021;128:740-53.

5. Sun J, Qian X, Zhang F, et al. HDAC6 inhibitor WT161 induces apoptosis in retinoblastoma cells and synergistically interacts with cisplatin. Transl Cancer Res 2019;8:2759-68.

6. Li H. Applications of Traditional Chinese Medicine in Antiviral and Anticancer Drug Development: An Interview with Dr. Yung-Chi (Tommy) Cheng, PhD. Yale J Biol Med 2020;93:381-4.

7. Zhou Z, Chen B, Chen S, et al. Applications of Network Pharmacology in Traditional Chinese Medicine Research. Evid Based Complement Alternat Med 2020;2020:1646905.

8. Aksorn N, Losuwannarak N, Tungsukruthai S, et al. Analysis of the Protein-Protein Interaction Network Identifying c-Met as a Target of Gigantol in the Suppression of Lung Cancer Metastasis. Cancer Genomics Proteomics 2021;18:261-72.

9. Losuwannarak N, Maiuthed A, Kitkumthorn N, et al. Gigantol Targets Cancer Stem Cells and Destabilizes Tumors via the Suppression of the PI3K/AKT and JAK/ STAT Pathways in Ectopic Lung Cancer Xenografts. Cancers (Basel) 2019;11:2032.

10. Zhao M, Sun Y, Gao Z, et al. Gigantol Attenuates the Metastasis of Human Bladder Cancer Cells, Possibly Through Wnt/EMT Signaling. Onco Targets Ther 2020;13:11337-46.

11. Yu $S$, Wang Z, Su Z, et al. Gigantol inhibits $W n t / \beta$-catenin signaling and exhibits anticancer activity in breast cancer cells. BMC Complement Altern Med 2018;18:59.

12. Andrade-Tomaz M, de Souza I, Rocha CRR, et al. The Role of Chaperone-Mediated Autophagy in Cell Cycle Control and Its Implications in Cancer. Cells 2020;9:2140.

13. Dang F, Nie L, Wei W. Ubiquitin signaling in cell cycle control and tumorigenesis. Cell Death Differ

Cite this article as: Zhang Y, Zhou L, Wang S, Wang M, Wu S. Exploration of retinoblastoma pathogenesis with bioinformatics. Transl Cancer Res 2021;10(7):3527-3537. doi: $10.21037 /$ tcr-21-1034
2021;28:427-38.

14. Moiseeva TN, Qian C, Sugitani N, et al. WEE1 kinase inhibitor AZD1775 induces CDK1 kinase-dependent origin firing in unperturbed G1- and S-phase cells. Proc Natl Acad Sci U S A 2019;116:23891-3.

15. Zhao S, Wang B, Ma Y, et al. NUCKS1 Promotes Proliferation, Invasion and Migration of Non-Small Cell Lung Cancer by Upregulating CDK1 Expression. Cancer Manag Res 2020;12:13311-23.

16. Lu S, Sun C, Chen H, et al. Bioinformatics Analysis and Validation Identify CDK1 and MAD2L1 as Prognostic Markers of Rhabdomyosarcoma. Cancer Manag Res 2020;12:12123-36.

17. Wang L, Qi Y, Wang X, et al. ECHS1 suppresses renal cell carcinoma development through inhibiting mTOR signaling activation. Biomed Pharmacother 2020;123:109750.

18. Wang Z, Li TE, Chen M, et al. miR-106b-5p contributes to the lung metastasis of breast cancer via targeting $\mathrm{CNN} 1$ and regulating Rho/ROCK1 pathway. Aging (Albany NY) 2020;12:1867-87.

19. Liu Y, Liang G, Zhou T, et al. Silencing UHRF1 Inhibits Cell Proliferation and Promotes Cell Apoptosis in Retinoblastoma Via the PI3K/Akt Signalling Pathway. Pathol Oncol Res 2020;26:1079-88.

20. Kim JK, Kan G, Mao Y, et al. UHRF1 downmodulation enhances antitumor effects of histone deacetylase inhibitors in retinoblastoma by augmenting oxidative stress-mediated apoptosis. Mol Oncol 2020;14:329-46.

21. Si X, Xu F, Xu F, et al. CADM1 inhibits ovarian cancer cell proliferation and migration by potentially regulating the PI3K/Akt/mTOR pathway. Biomed Pharmacother 2020;123:109717.

22. Saito M, Goto A, Abe N, et al. Decreased expression of CADM1 and CADM4 are associated with advanced stage breast cancer. Oncol Lett 2018;15:2401-6.

23. Niu X, Nong S, Gong J, et al. MiR-194 promotes hepatocellular carcinoma through negative regulation of CADM1. Int J Clin Exp Pathol 2020;13:1518-28.

(English Language Editors: J. Collie and J. Gray) 